\title{
Pathophysiology and management of sensitive skin: position paper from the special interest group on sensitive skin of the International Forum for the Study of Itch (IFSI)
}

\author{
L. Misery, ${ }^{1, *}$ (D) E. Weisshaar, ${ }^{2}$ E. Brenaut, ${ }^{1}$ (D) A.W.M. Evers, ${ }^{3}$ F. Huet, ${ }^{1}$ S. Ständer, ${ }^{4}$ (D) A. Reich, ${ }^{5}$ \\ E. Berardesca, ${ }^{6}$ (D E. Serra-Baldrich, ${ }^{7}$ J. Wallengren, ${ }^{8}$ D. Linder, ${ }^{9}$ (D) J.W. Fluhr, ${ }^{10}$ \\ J.C. Szepietowski, ${ }^{11}$ iD H. Maibach, ${ }^{12}$ for the Special Interest Group on sensitive skin of the International \\ Forum for the Study of Itch (ISFI) ${ }^{\dagger}$ \\ ${ }^{1}$ Department of Dermatology, University Hospital of Brest, Brest, France \\ ${ }^{2}$ Department of Dermatology, Occupational Dermatology, Ruprecht-Karls University Heidelberg, Heidelberg, Germany \\ ${ }^{3}$ Department of Health, Medical and Neuropsychology, Faculty of Social and Behavioral Science, Leiden, The Netherlands \\ ${ }^{4}$ Department of Dermatology, Center for Chronic Pruritus, University Hospital Münster, Münster, Germany \\ ${ }^{5}$ Department of Dermatology, University of Rzeszow, Rzeszów, Poland \\ ${ }^{6}$ San Gallicano Dermatological Institute, Rome, Italy \\ ${ }^{7}$ Department of Dermatology, Hospital de la Santa Creu i Sant Pau, Barcelona, Spain \\ ${ }^{8}$ Department of Dermatology and Venereology, Skåne University Hospital, Lund, Sweden \\ ${ }^{9}$ Section of Biostatistics, University of Oslo, Oslo, Norway \\ ${ }^{10}$ Department of Dermatology and Allergy, Charité-Universitätsmedizin Berlin, Berlin, Germany \\ ${ }^{11}$ Department of Dermatology, Venereology and Allergology, Wroclaw Medical University, Wroclaw, Poland \\ ${ }^{12}$ Department of Dermatology, School of Medicine, University of California, San Francisco, San Francisco, CA, USA \\ *Correspondence: L. Misery. E-mail: laurent.misery@chu-brest.fr
}

\begin{abstract}
The special interest group on sensitive skin of the International Forum for the Study of Itch previously defined sensitive skin as a syndrome defined by the occurrence of unpleasant sensations (stinging, burning, pain, pruritus and tingling sensations) in response to stimuli that normally should not provoke such sensations. This additional paper focuses on the pathophysiology and the management of sensitive skin. Sensitive skin is not an immunological disorder but is related to alterations of the skin nervous system. Skin barrier abnormalities are frequently associated, but there is no cause and direct relationship. Further studies are needed to better understand the pathophysiology of sensitive skin - as well as the inducing factors. Avoidance of possible triggering factors and the use of well-tolerated cosmetics, especially those containing inhibitors of unpleasant sensations, might be suggested for patients with sensitive skin. The role of psychosocial factors, such as stress or negative expectations, might be relevant for subgroups of patients. To date, there is no clinical trial supporting the use of topical or systemic drugs in sensitive skin. The published data are not sufficient to reach a consensus on sensitive skin management. In general, patients with sensitive skin require a personalized approach, taking into account various biomedical, neural and psychosocial factors affecting sensitive skin.

Received: 1 July 2019; Accepted: 20 September 2019

\section{Conflicts of interest}

LM is an consultant of Beiersdorf, Bioderma, Clarins, Expanscience, Johnson \& Johnson, Nestlé Skin Health, Pierre Fabre, La Roche-Posay, Solabia and Uriage; EW, AWME, SS, EB, ESB, JW, DL, JCS and HM declared none; EB is an consultant of Bioderma, Clarins, Nestlé Skin Health and Pierre Fabre; FH is an consultant of Beiersdorf, Clarins and Pierre Fabre; AR is an consultant of Bioderma, Galderma and Pierre Fabre; JWF is an consultant of Bioderma, Nestlé Skin Health, Sebapharma and Pierre Fabre.
\end{abstract}

\section{Funding source}

None.

\footnotetext{
†Other members: Golara Honari (Stanford University School of Medicine, Department of Dermatology, Redwood City, USA), Christelle Le Gall-lanotto (University Hospital of Brest, Department of Dermatology, Brest, France), Kenji Takamori (Juntendo University Urayasu Hospital, Department of Dermatology, Chiba, Japan), Renée Richters (Radboud University Medical Center, Department of Dermatology, Nijmegen, The Netherlands).
} 


\section{Introduction}

Although usually not severe, sensitive skin is a common health problem. The self-declared prevalence of sensitive skin affects approximately $60-70 \%$ of women and $50-60 \%$ of men. ${ }^{1}$

Itch is one of major symptoms of sensitive skin. Thus, the International Forum for the Study of Itch (IFSI) decided during the 7th World Congress on Itch (Boston, 2013) to initiate a special interest group (SIG) on this topic. Members of the SIG were selected according to their interest and expertise on itch and/or sensitive skin. The group comprises dermatologists, psychologists and biologists from different countries. All SIG members are academics, and none is employed by a cosmetic company. Conflicts of interest are provided below.

Using the Delphi method (five rounds), sensitive skin was defined as 'a syndrome defined by the occurrence of unpleasant sensations (stinging, burning, pain, pruritus and tingling sensations) in response to stimuli that normally should not provoke such sensations. These unpleasant sensations cannot be explained by lesions attributable to any skin disease. The skin can appear normal or be accompanied by erythema. Sensitive skin can affect all body locations, especially the face'. ${ }^{2}$

This additional paper focuses on the pathophysiology and the management of sensitive skin. Independent reviews ${ }^{3-13}$ and one book (two editions) $)^{14,15}$ were previously published on this topic. Nonetheless, there is a need for a consensual approach after the publication of the consensual definition. This work was performed over 2 years [from the 9th World Congress on Itch (Wroclaw, 2017) until the 10th World Congress on Itch (Sydney, 2019)] by frequent e-mail discussions and several face-to-face meetings.

\section{Methods}

The methodology has been adopted from our previous work on the definition ${ }^{2}$ and a recent work on discoid lupus erythematosus. ${ }^{16}$ We employed the Delphi method, which is a consensus building using a series of questionnaires by a panel of selected experts. ${ }^{17,18}$ This iterative approach allows convergence towards a consensus. Summary statistics of survey results are shared after each questionnaire round. The experts are encouraged to revise their answers in light of responses from other panel members. During this process, the range of answers decreases as the group converges towards consented agreements regarding the different items.

The first step was split into two phases: item generation followed by item reduction. An in-person approach was used for item generation, whereas Internet-based Delphi consensus process and a face-to-face meeting were used for item reductions.

The item generation process allowed to initiate the following list of 37 items:

- Pathophysiology: stratum corneum, nervous system, neurogenic inflammation, TRPV1 (transient receptor potential vanilloid 1), environmental factors, UV, cosmetics, symptoms, sensations, inflammation, innervation, nervous system, neurosensory irritation, barrier function, sensitivity, itch, erythema, non-visible irritation, hydration, small nerve fibre, stratum corneum hydration, skin nervous system, burning, exacerbating environmental factors, xerosis, TRP (transient receptor potential), atopy, neuropathy, sensor proteins.

- Management: allergen, irritant, moisturizer, photoprotection, tolerability, emollients, UV protection, avoidance of triggers, inhibition of neurogenic inflammation.

For item reduction, the list of 37 items was subjected to one round of an Internet-based Delphi process using standard methodology with the aim of a $75 \%$ consensus. A face-to-face meeting was organized (Paris, 14 September 2018, on the occasion of the EADV congress), and 10 members of the expert panel built a list of 12 items (pathophysiology: 7; management: 5). Finally, a second round of an Internet-based Delphi process was organized and the list was approved by $17 / 17$ voters. The addition of nocebo and placebo effects was proposed a few weeks later and approved by all voters. The final list of 14 items was made of eight items on pathophysiology (skin barrier function, stratum corneum, atopic dermatitis and disposition, environmental factors, sensory proteins, epidermal nerve endings, vasculature, stress, nocebo effect) and six on management (avoidance of triggers, emollients/moisturizers, photoprotection, inhibition of neurogenic inflammation, placebo effect, holistic approach).

A list of members of the group for writing a synthesis of the bibliographical data and for preparing proposed recommendations was composed during the Paris meeting. The contributions were given to all members of the group for online discussions and a face-to-face meeting (Washington, 2 March 2019, on the occasion of the AAD congress). LM synthesized them for this paper, then the paper was reviewed by HM and all SIG members approved it after discussions.

The recommendations were discussed and rewritten during the Washington meeting (seven participants) and subsequently discussed and approved by 17/17 members after a 2-round Internet-based Delphi process. The recommendations are given in Table 1.

\section{Pathophysiology of sensitive skin}

\section{Relationship with skin barrier function}

The skin barrier has multiple functions and protects us from external threats such as chemicals, infectious agents and allergens. ${ }^{19}$ The reported symptoms of sensitive skin cannot be allocated to epidermal functioning and/or epidermal structures. Little is known about sensitive skin and its relation to skin barrier function. It can be postulated that sensitive skin may be associated with, e.g. increased permeability of the skin barrier, dry skin, hampered functioning by skin diseases like atopic 
Table 1 Recommendations from the special interest group on sensitive skin of the International Forum for the Study of Itch

1. The avoidance of possible triggering factors and the use of welltolerated cosmetics, especially those containing inhibitors of unpleasant sensations, might be suggested for patients with sensitive skin

2. There is no clinical trial supporting the use of topical or systemic drugs in sensitive skin

3. There is no study providing data to reach a consensus on the management of sensitive skin

dermatitis $(\mathrm{AD})$ and rosacea, or it may be associated with increased trans-epidermal water loss and decreased amount of natural moisturizing factor. ${ }^{20-22}$ Sensitive skin is reported to be associated with $\mathrm{AD}$ severity but not necessarily with barrier condition. $^{22}$ Others published that barrier disruption is associated with itch but no clear information is provided to which extent this is associated with sensitive skin. ${ }^{21}$ These symptoms may also be caused by the fact that there is a significant interplay between genetic and environmental factors not only in the pathogenesis of $\mathrm{AD}$ but also in the pathogenesis of sensitive skin and its symptoms. ${ }^{23}$ In summary, the SIG concludes that there is limited research on the relation of sensitive skin and barrier function.

\section{Role of stratum corneum}

Subjects with sensitive skin have been reported to have a thinner stratum corneum with a reduced corneocyte number causing a higher transcutaneous penetration of water-soluble chemicals. ${ }^{24}$ Frosch and Kligman, ${ }^{25}$ by testing different irritants, showed a $14 \%$ prevalence of sensitive skin in the normal population. They suggested that sensitive to be correlated with a thin permeable stratum corneum which makes these subjects more susceptible to chemical irritation. Moreover, the declined barrier function in sensitive skin has already been reported as the result of an imbalance of intercellular lipids of the stratum corneum. ${ }^{26}$ Although impaired barrier function could be a mechanism of sensitive skin, other factors are also possible mechanisms such as changes in the nerve system and/or the structure of the epidermis. Yokota et al. ${ }^{27}$ classified sensitive skin into three types based on their physiological parameters: type I (low barrier function group), type II (inflammation group with normal barrier function and inflammatory changes) and type III (pseudohealthy group in terms of normal barrier function and no inflammatory changes). In all types, a high content of nerve growth factor has been observed in the stratum corneum, relative to that of non-sensitive skin. Both in type II and type III, the sensitivity to electrical stimuli was high. Yamasaki and Gallo ${ }^{28}$ proposed that the innate immune system, especially cathelicidin, could trigger an abnormal inflammatory reaction that mediates the symptoms of rosacea and sensitive skin.

Changes in stratum corneum thickness and therefore of transcutaneous penetration may explain regional differences in specific areas of sensitive skin. The face has demonstrated to be the most common site of skin sensitivity, which may be due to the larger number of products used on the face (particularly in women), a thinner barrier in facial skin and a greater density of nerve endings. ${ }^{29}$ The nasolabial fold was reported to be the most sensitive region of the facial area, followed by the malar eminence, chin, forehead and upper lip. ${ }^{30,31}$ Hands, scalp, feet, neck, torso and back sensitivity follow facial sensitivity in descending order of prevalence. ${ }^{32}$ Significant numbers of individuals experience sensitivity of the scalp. ${ }^{8,33}$ From an instrumental viewpoint, stratum corneum of subjects with sensitive skin is frequently associated with increased TEWL, as shown in several studies confirmed in a systematic literature review, ${ }^{8}$ but the results remain controversial. The hypothesis of a reduced content of ceramides in the stratum corneum has been suggested. ${ }^{34}$ No significant differences have been confirmed in sebum content and skin $\mathrm{pH}^{8}$ even though a high $\mathrm{pH}$, particularly in topically applied products could increase transcutaneous penetration of topically applied chemicals and elicit reactions associated with sensitive skin. ${ }^{35}$

\section{Relationship with atopic dermatitis and disposition}

Although the definition of sensitive skin indicates that the unpleasant sensations related to sensitive skin cannot be explained by lesions attributable to any skin disease, ${ }^{2}$ patients with $\mathrm{AD}$ were previously investigated in the frequency and extent of sensitive skin. ${ }^{22}$ It was reported that sensitive skin is more common in $\mathrm{AD}$ patients than in healthy subjects. ${ }^{36} \mathrm{~A}$ large proportion of adults with sensitive skin reported $\mathrm{AD}$ in their medical history during childhood (this fact is suggestive for common factors between sensitive skin and AD). Sensitive skin was also reported to be frequent in extrinsic $\mathrm{AD}$ and correlates with disease severity markers but not necessarily with skin barrier impairment. ${ }^{37}$ Extrinsic $\mathrm{AD}$ patients, possessing higher barrier impairment than intrinsic $\mathrm{AD}$, showed a significantly higher frequency of positive lactic acid stinging test (LAST) than did intrinsic $\mathrm{AD}$ patients. ${ }^{38}$ However, the relationship between each sensory symptom of sensitive skin and AD severity/barrier markers remains unclear. Whereas some consider $\mathrm{AD}$ as an aetiology of sensitive skin, $\mathrm{AD}$ is rather a major confounder with sensitive skin than a causal factor, ${ }^{39}$ meaning that patients with $\mathrm{AD}$ or sensitive skin are probably frequently mixed up.

\section{Role of environmental factors}

The role of the environmental factors on sensitive skin has been mainly explored through patient interviews. A recent literature review with a meta-analysis analysed the role of environmental factors on sensitive skin. ${ }^{40}$ Thirteen studies were included representing 20486 subjects. These subjects were recruited in different ways: random sampling, opportunity sampling or stratified sampling. Questionnaires about triggering factors of sensitive skin were fulfilled during face-to-face meetings, phone 
interviews or web surveys. Subjects were classified in groups 'sensitive skin' or 'no sensitive skin', and the triggering factors (not aggravating factors) were searched based on different questions (e.g. 'Is your facial skin easily irritated by ...' or 'Do you suffer from burning, prickling or irritation in the presence of ..?').

According to the patient-reported outcome, sensitive skin could be triggered by several factors. The odds ratios of this meta-analysis are presented in Table 2. Notably, there is only one exposure study, which showed a role of hair dyes. ${ }^{41}$ Hence, the probable role of environmental factors on sensitive skin is mainly assessed by the patient's point of view until now. There is no proof on the involvement of these factors, and further studies are needed.

\section{Biochemical role of sensory proteins, including TRP}

The triggering of sensitive skin by physical, chemical or physicochemical is surprising. It can be explained by the activation or rather the hyper-activation of proteins present on the surface of keratinocytes and intra-epidermal nerve endings. Their function is to allow the perception of multiple environmental factors. Especially involved are receptors from the family of transient receptor proteins (TRPs) ${ }^{42,43}$ and others. These proteins can be activated both by physical and chemical factors without being able to distinguish between the two. For example, TRPV1 can be activated by both capsaicin and protons or heat, while TRPM8 can be activated by both cold and menthol. In sensitive skin, sensory proteins such as TRPV1 and ASIC3 have been reported to be overexpressed. ${ }^{44}$

\section{Role of epidermal nerve endings}

Because sensitive skin is mainly characterized by a wide variety of sensory symptoms, it is likely that a neurosensory dysfunction in the skin represents one of the pathological mechanisms of

Table 2 Triggering factors of sensitive skin suggested by interviewees in surveys

\begin{tabular}{lll}
\hline Factor & Odds ratio & 95\% IC \\
\hline Cosmetic & 7.12 & $3.98-12.72$ \\
Wet air & 3.83 & $2.48-5.91$ \\
Air conditioning & 3.60 & $2.11-6.14$ \\
Temperature variation & 3.53 & $2.69-4.63$ \\
Heat & 3.50 & $2.56-4.77$ \\
Water & 3.46 & $2.82-4.25$ \\
Pollution & 3.18 & $2.37-4.27$ \\
Dry air & 3.04 & $2.22-4.16$ \\
Cold & 2.73 & $1.94-3.84$ \\
Wind & 2.33 & $1.69-3.22$ \\
Sun & 1.81 & $1.61-2.04$ \\
Emotion & 1.77 & $1.44-2.17$ \\
\hline
\end{tabular}

Comparison between people with sensitive skin and healthy subjects. Results from a meta-analysis. sensitive skin. ${ }^{9}$ Without excluding other possible explanations, the role of the nervous system in the development of sensitive skin is crucial and a growing body of evidences supporting this hypothesis. $^{4,8,11}$ Skin hypersensitivity (as assessed by the Sensitive Scale) is more severe in patients with characteristics of neuropathic pain using the DN-4 (Douleur neuropathique, french name for neuropathic pain) questionnaire ${ }^{45,46}$ or the Neuropathic Pain Sensory Inventory (NPSI). ${ }^{46}$ In a recent epidemiological study on 5000 volunteers, very sensitive skin was twice as common in people with irritable bowel syndrome, ${ }^{47}$ considered as secondary to peripheral nervous system alterations. Reciprocally, irritable bowel syndrome was more frequent in sensitive skin and associated with increased severity. Furthermore, an immunohistochemical study compared 50 biopsies of subjects with or without sensitive skin. ${ }^{48}$ The number of intra-epidermal nerve fibres was significantly decreased, revealing that the $\mathrm{A} \delta$ or C fibre population was altered. In a case-control study, 21 subjects with and 21 subjects without sensitive skin were compared with a significant decrease in the heat-pain threshold detection in the sensitive skin group compared with the control group. ${ }^{46}$ Conversely, vibration and cold threshold detection revealed no difference. These findings are similar to those observed in smallfibre neuropathies. ${ }^{49-51}$ The reduction in intra-epidermal nerve fibres and alterations of $\mathrm{C}$ fibres can induce hyper-reactivity of the remaining nerve endings, which can explain both sensitive skin and small-fibre neuropathy symptoms.

\section{Role of vasculature}

Long-standing irritant exposure may be associated with erythema. But even patients without erythema or other signs of visible inflammation display hyper-reactivity of dermal blood vessels which can be measured by laser doppler velocimetry (LDV) or assessing skin colour with a chromameter. ${ }^{11,52}$ Hence, testing of vasodilation of the skin may be an objective approach to test sensitive skin. Methyl nicotinate applied to the upper third of the ventral forearm for $15 \mathrm{~s}$ induces a strong vasodilation which can be measured with LDV. Increased vascular reaction to methyl nicotinate was reported in subjects with sensitive skin compared with normal subjects. ${ }^{53}$ Similar analysis can be performed following application of various concentrations of sodium lauryl sulphate. ${ }^{11}$

Histamine has been suggested as a vasodilator in sensitive skin when the cumulative lactic acid sting scores were compared with the histamine itch scores in 32 young subjects: all subjects who were stingers were also moderate to intense itchers, whereas $50 \%$ of the moderate itchers showed little or no stinging response. ${ }^{7}$ Other mediators could contribute to the hyper-reactivity of blood vessels. ${ }^{54}$ Dermal blood vessels of the extremities are controlled by the sympathetic nervous system, whereas those of the head and upper thorax (the blushing area) are predominantly controlled by the parasympathetic nervous system and by circulating vasoactive agents. It may explain why the results of 
experimental tests on the forearm may differ from what can be obtained on the facial skin. Parasympathetic innervation of blood vessel walls regulates vasodilation. In addition to acetylcholine, neuropeptides as VIP (vasoactive intestinal peptide), PACAP (pituitary adenylate cyclase-activating peptide) and NPY (neuropeptide Y) control these reactions. ${ }^{55}$ Upon intradermal injection into human skin, these neuropeptides induce vasodilation, which can be partially inhibited by repeated pretreatment with capsaicin inducing tachyphylaxis. ${ }^{54}$

\section{Role of stress}

There is a long-standing history of the idea that stress plays a substantial role in the skin. Moreover, there are clear evidences that the symptom of itch can be triggered only by negative emotions, e.g. after watching a threatening movie. ${ }^{56}$ Due to possible central sensitivity and interaction at different response levels, these factors might be equally relevant for the sensitive skin symptoms, at least during stressful periods or for people with high stress levels.

\section{Nocebo effect}

Whether one responds or not to a given treatment option or develops severe symptoms or side effects is strongly affected by the expectation of the patients about the treatment or condition, as demonstrated from nocebo research. ${ }^{57-59}$ Nocebo effects - the opposite to placebo effects - can be described as negative treatment consequences, not only due to the treatment mechanisms, but also due to the negative expectations of the patient. ${ }^{57-59}$ In sensitive skin, there is relatively consistent evidence for the relevance of nocebo effects from related fields, such as itch and skin responses. ${ }^{60}$ Several studies showed that negative (catastrophic) suggestions, in combination with or without conditioning, results in more itch or larger wheal size in healthy subjects. ${ }^{61-63}$ Nocebo effects in the clinical setting have been examined in outpatients who previously showed adverse drug reactions. About $27 \%$ of patients who were exposed to placebo medication as part of routine exposure protocol displayed nocebo responses, such as itch and skin lesions, after the placebo administration. ${ }^{64}$

\section{Management of sensitive skin}

\section{Avoidance of triggers}

Although there is no confirmatory study in the literature, it is probably indispensable to avoid the supposed triggering factors of sensitive skin. Since only exposure study showed a role of hair dyes on sensitive scalp and facial sensitive skin, ${ }^{41,65}$ hair dyes should be avoided, in particular those containing ammonia. In a recent literature review and meta-analysis, cosmetics were the main triggering factors of sensitive skin according to the patientreported outcome. ${ }^{40}$ The presence of potentially irritating substances in their composition (alpha-hydroxy acids, propylene glycol, alcohol fragrances, preservatives, surfactants and others) increases the possibility of the occurrence of symptoms. The main advice is to limit the use of cosmetics or to use high tolerance products that contain little or no preservatives and surfactants, as well as no fragrances. Some cosmetics are supposed to contain active soothing ingredients which may be anti-inflammatory or exhibiting inhibitory effects on neurogenic inflammation. Comparative tests should be carried out. In this literature review, ${ }^{40}$ physical factors were also frequently declared by patients as aggravating or triggering factors of sensitive skins: wet air, variations in temperature, air conditioning, heat, dry air, cold, wind and sun should be avoided by adjusting the habits of life, profession and leisure.

\section{Emollients/moisturizers}

Many people (women more frequently than men) report to have sensitive skin which is often associated with the use of a skin moisturizer or an emollient. ${ }^{66}$ Nonetheless, the treatment of dry skin by moisturizing the stratum corneum could be useful since dry skin may be associated with sensitive skin symptoms. Depending on the texture and the skin type, no definite recommendation for treating sensitive skin can be provided. ${ }^{66,67}$ When it comes to emollients and moisturizers, it is generally recommended to have as few ingredients as possible, but according to current regulations not all ingredients are documented on the package of the product. Cleansing of the skin should be performed in a mild way using, for example cleaning lotions. When it comes to treating sensitive skin, symptoms should be reduced, but it is not clear to which extent they are associated with sensitive skin and its treatment. It was reported that emollients can substantially reduce itching even after the first administration, restore skin barrier function and are considered as an approach to sensitive skin in eczema. ${ }^{68}$ Shower creams and lotions with skin-similar lipids, also used in combination, were reported to restore skin barrier function, increase skin hydration and become an effective skin moisturizing option for patients with atopic dry skin which is also associated with sensitive skin. ${ }^{69}$

Occupational skin diseases like irritant contact dermatitis are frequently associated with sensitive skin. In affected patients, prevention is an important issue. Several ways of protecting the barrier by prevention have been published, including protecting the skin by wearing gloves, using protective creams and regular moisturizing the skin. ${ }^{70}$ This may prevent the development of sensitive skin.

\section{Photoprotection}

Ultraviolet radiation (UVR) exerts a wide spectrum of deleterious actions on the skin. ${ }^{71}$ Many of these phenomena may augment symptoms of sensitive skin upon sun exposure. For instance, UVR increases the release of neuropeptides from nerve endings such as substance $\mathrm{P}$ and calcitonin gene-related peptide. Both of them are known to induce vasodilatation, itch or 
burning pain sensations and neuroinflammation. ${ }^{72}$ UVR also downregulates $\beta$-endorphin and enkephalin levels in the skin which may reduce the painful sensations and increases the level of proinflammatory cytokines, such as interleukin (IL) $1 \alpha$, IL10 , IL-33 or tumour necrosis factor $\alpha^{72}$ In addition, UVR also elicits sensitization of nociceptors. ${ }^{73}$ Increased epidermal permeability upon UVR due to changes in epidermal structure and basal membrane damage increases the chance of deeper penetration of potentially irritating factors into the skin. UVR also leads to angiogenesis and formation of erythema.

All these above-mentioned phenomena unanimously indicate the need of proper sun protection among people suffering from sensitive skin. Thus, subjects with sensitive skin should limit sun exposure, seek shade while being outside, wear photoprotective clothing and proper headgear such as a wide brimmed hat and apply sunscreens. Patients with sensitive skin should apply sunscreens with a sun protection factor of at least 30, which also provide broad-spectrum sun protection indicating that the formulations contain UVA filters, as these products provide better protection from sun damage. ${ }^{74}$ Nonetheless, there is a need to remember that sunscreens could be also inducers of sensitive skin.

Importantly, no single protective measure usually ensures sufficient UVR protection and thus combining multiple sun protection behaviours may be needed to achieve optimal sun protection. $^{75}$

\section{Inhibition of neurogenic inflammation}

The key players in neurogenic inflammation in sensitive skin are numerous. ${ }^{9}$ These pruritogens target dermal and epidermal nerves which are subject to changes in the morphological nerve endings with subsequent hyperactivity and neurogenic inflammation. ${ }^{76}$ These pruritogens act via different receptors and ion channels such as TRPA1 and TRPV1. TRPV1 is an important ion channel on epidermal nerve fibres which mediates neurogenic inflammation. It is also expressed on non-neuronal cells such as keratinocytes, mast cells or Langerhans cells and has a role in barrier disturbance. ${ }^{77}$

Substances addressing the TRPV1, such as its agonist capsaicin, have a less beneficial side-effect profile and may worsen neurogenic inflammation and sensitive skin. However, one study demonstrated the successful use of a novel topical TRPV1 agonist in 30 patients with sensitive skin. ${ }^{78}$ The association of inhibitors of neurogenic inflammation might be valuable. ${ }^{79}$

Alternatively, low level laser/light therapy ${ }^{80,81}$ and botanical anti-inflammatories ${ }^{82}$ have shown beneficial effects in sensitive skin.

\section{Placebo effect}

Communication about treatment and prognosis is particularly relevant in the case of sensitive skin, where patients and professionals are confronted with the lack of diagnostic criteria and limited treatment options. This frequently results in a feeling of insecurity and ambiguity for both professionals and patients. Communication about a treatment or prognosis and prior expectations of patients and professionals regarding treatment are the most essential mechanisms of placebo effects. ${ }^{59,83,84} \mathrm{Pla}$ cebo effects can be defined as beneficial treatment effects that cannot be ascribed to the treatment mechanisms itself, but that is a consequence of the expectation of patients. ${ }^{59,83,84}$

Although there is no study that directly focuses on the role of placebo effects in sensitive skin, there is indirect evidence from research on placebo effects in itch. For example, placebo effects for itch can be induced by verbal suggestions or a combination of verbal suggestions and conditioning. ${ }^{63}$ This type of instructions or verbal suggestions about possible treatment effects, seems also to work, e.g. for expectancies about itch decrease, when participants are informed about the fact that they receive a placebo treatment. ${ }^{85}$ However, the combination of verbal suggestions and more automatic processes of conditioning is usually most effective. ${ }^{63}$ During conditioning, people learn that a specific context or stimulus (e.g. colour or cream) is consistently associated with a decrease or increase in itch. Evidence is quite consistent for various physical symptoms, such as itch and pain. $^{83,84}$

Clinical recommendations emphasize the importance to make maximal use of the placebo effects in clinical practice, particularly for patients where hardly any effective treatment options exist, for example to train clinicians in relevant communication skills. ${ }^{59}$ In view of the unclear diagnosis and therapeutical options for sensitive skin, it might be particularly important to study these mechanisms and treatment options of placebo effects for people with sensitive skin.

\section{Conclusion: holistic approach}

As the pathogenesis of sensitive skin is not clearly understood and most probably is of multifactorial origin, there is no 'gold standard' of treatment for sensitive skin. It is necessary to underline that the literature data on the therapy of sensitive skin are limited and the presented results of the studies are difficult to compare due to different understanding of the nature of sensitive skin, variations in populations studied and various methodology employed. Many of the above-mentioned treatment modalities are extrapolated from other studies and suggested by the experts. Therefore, the bias could not be completely excluded.

In general, it seems clear that patients with sensitive skin require a personalized approach, taking into account the various possible biomedical, neural and psychosocial factors. The avoidance of exacerbating factors, restoration of damaged skin barrier, photoprotection and anti-inflammatory agents as well as possible triggering psychosocial factors should be considered. Many patients could also benefit from psychological support, which could help them to cope with the problem of sensitive skin. 


\section{References}

1 Farage MA. The prevalence of sensitive skin. Front Med 2019; 6: 98.

2 Misery L, Ständer S, Szepietowski JC et al. Definition of sensitive skin: an expert position paper from the special interest group on sensitive skin of the international forum for the Study of Itch. Acta Derm Venereol 2017; 97: 4-6.

3 Duarte I, Silveira JEPS, Hafner MFS et al. Sensitive skin: review of an ascending concept. An Bras Dermatol 2017; 92: 521-525.

4 Misery L. Sensitive skin. Expert Rev Dermatol 2013; 8: 631-637.

5 Misery L, Loser K, Ständer S. Sensitive skin. J Eur Acad Dermatol Venereol 2016; 30(Suppl 1): 2-8.

6 Inamadar AC, Palit A. Sensitive skin: an overview. Indian J Dermatol Venereol Leprol 2013; 79: 9-16.

7 Berardersca E, Farage MA, Maibach H. Sensitive skin: an overview. Int J Cosmet Sci 2011; 35: 2-8.

8 Richters R, Falcone D, Uzunbajakava $\mathrm{N}$ et al. What is sensitive skin ? A systematic literature review of objective measurements. Skin Pharmacol Physiol 2015; 28: 75-83.

9 Stander S, Schneider SW, Weishaupt C et al. Putative neuronal mechanisms of sensitive skin. Exp Dermatol 2009; 18: 417-423.

10 Farage MA. Perceptions of sensitive skin: changes in perceived severity and associations with environmental causes. Contact Dermatitis 2008; 59: 226-232.

11 Farage MA, Katsarou A, Maibach HI. Sensory, clinical and physiological factors in sensitive skin: a review. Contact Dermatitis 2006; 55: 1-14.

12 Farage MA, Maibach HI. Sensitive skin: closing in a physiological cause. Contact Dermatitis 2010; 62: 137-149.

13 Maibach HI, Lammintausta K, Berardesca E et al. Tendency to irritation: sensitive skin. J Am Acad Dermatol 1989; 21: 833-835.

14 Berardesca E, Fluhr JW, Maibach HI. Sensitive Skin Syndrome. Taylor \& Francis, New York, NY, 2006.

15 Honari G, Andersen RM, Maibach HI. Sensitive Skin Syndrome, 2nd edn. CRC Press, Boca Raton, FL, 2017.

16 Elman SA, Joyce C, Nyberg F et al. Development of classification criteria for discoid lupus erythematosus: results of a Delphi exercise. J Am Acad Dermatol 2017; 77: 261-267.

17 Diamond IR, Grant RC, Feldman BM et al. Defining consensus: a systematic review recommends methodologic criteria for reporting of Delphi studies. J Clin Epidemiol 2014; 67: 401-409.

18 Graham B, Regehr G, Wright JG. Delphi as a method to establish consensus for diagnostic criteria. J Clin Epidemiol 2003; 56: 1150-1156.

19 Angelova-Fischer I. Irritants and Skin Barrier Function. Curr Probl Dermatol 2016; 49: 80-89.

20 Proksch E, Weidinger S. New insights into the pathogenesis of sensitive skin. Hautarzt 2011; 62: 900-905.

21 Rerknimitr P, Otsuka A, Nakashima C et al. The etiopathogenesis of atopic dermatitis: barrier disruption, immunological derangement, and pruritus. Inflamm Regen 2017; 37: 14.

22 Yatagai T, Shimauchi T, Yamaguchi $\mathrm{H}$ et al. Sensitive skin is highly frequent in extrinsic atopic dermatitis and correlates with disease severity markers but not necessarily with skin barrier impairment. J Dermatol Sci 2018; 89: 33-39.

23 Otsuka A, Nomura T, Rerknimitr P et al. The interplay between genetic and environmental factors in the pathogenesis of atopic dermatitis. Immunol Rev 2017; 278: 246-262.

24 Berardesca E, Cespa M, Farinelli N et al. In vivo transcutaneous penetration of nicotinates and sensitive skin. Contact Dermatitis 1991; 25: 35-38.

25 Frosch PJ, Kligman AM. A method of apraising the stinging capacity of topically applied substances. J Soc Cosmet Chem 1977; 28: 197-209.

26 Ohta M, Hikima R, Ogawa T. Physiological characteristics of sensitive skin classified by stinging test. J Cosmet Sci Soc Jpn 2000; 23: 163-167.

27 Yokota T, Matsumoto M, Sakamaki T et al. Classification of sensitive skin and development of treatment system appropriate for each group. IFSCC Mag 2003; 6: 303-307.
28 Yamasaki K, Gallo RL. The molecular pathology of rosacea. J Dermatol Sci 2009; 55: 77-81.

29 Chew A, Maibach H. Sensitive skin. In: Loden M, Maibach H, eds. Dry Skin and Moisturizers: Chemistry and Function. CRC Press, Boca Raton, FL, 2000: 429-440.

30 Marriott M, Whittle E, Basketter DA. Facial variations in sensory responses. Contact Dermatitis 2003; 49: 227-231.

31 Distante F, Bonfigli A, Rigano L et al. Intra- and inter-individual differences in facial skin biophysical properties. Cosmet Toilet 2002; 7: 149-158.

32 Saint-Martory C, Roguedas-Contios AM, Sibaud V et al. Sensitive skin is not limited to the face. Br J Dermatol 2008; 158: 130-133.

33 Misery L, Rahhali N, Ambonati M et al. Evaluation of sensitive scalp severity and symptomatology by using a new score. J Eur Acad Dermatol Venereol 2011; 25: 1295-1834.

34 Cho HJ, Chung BY, Lee HB et al. Quantitative study of stratum corneum ceramides contents in patients with sensitive skin. J Dermatol 2012; 39: 295-300.

35 Kim E, Kim S, Nam GW et al. The alkaline pH-adapted skin barrier is disrupted severely by SLS-induced irritation. Int J Cosmet Sci 2009; 31: 263-269.

36 Willis CM, Shaw S, de Lacharrière O et al. Sensitive skin: an epidemiological study. Br J Dermatol 2001; 145: 258-263.

37 Schmitt J, Langan S, Williams HC. What are the best outcome measurements for atopic eczema? A systemic review. J Allergy Clin Immunol 2007; 120: $1389-1398$.

38 Mori T, Ishida K, Mukumoto S et al. Comparison of skin barrier function and sensory nerve electric current perception threshold between IgE-high extrinsic and IgE-normal intrinsic types of atopic dermatitis. Br J Dermatol 2009; 162: 83-90.

39 Andersen RM, Thyssen JP, Maibach HI. Qualitative vs. quantitative atopic dermatitis criteria - in historical and present perspectives. J Eur Acad Dermatol Venereol 2016; 30: 604-618.

40 Brenaut E, Barnetche T, Le-Gall Ianotto C et al. Triggering factors in sensitive skin from the worldwide patient's point of view: A systematic literature review and meta-analysis. J Eur Acad Dermatol Venereol 2019. https://doi.org/10.1111/jdv.15985 [Epub ahead of print].

41 Bernard A, Houssin A, Ficheux AS et al. Consumption of hair dye products by the French women population: usage pattern and exposure assessment. Food Chem Toxicol 2016; 88: 123-132.

42 Boulais N, Misery L. The epidermis: a sensory tissue. Eur J Dermatol 2008; 18: $119-127$.

43 Caterina MJ, Pang Z. TRP channels in skin biology and pathophysiology. Pharmaceuticals (Basel) 2016; 9: E77.

44 Kim EJ, Lee DH, Kim YK et al. Adiponectin deficiency contributes to sensitivity in human skin. J Invest Dermatol 2015; 135: 2331-2334

45 Saint-Martory C, Sibaud V, Theunis J et al. Arguments for neuropathic pain in sensitive skin. Br J Dermatol 2015; 172: 1120-1121.

46 Huet F, Dion A, Batardière A et al. Sensitive skin can be small fibre neuropathy: results from a case-control quantitative sensory testing study. $\mathrm{Br}$ J Dermatol 2018; 179: 1157-1162.

47 Misery L, Duboc H, Coffin B et al. Association between two painful and poorly understood conditions: irritable bowel and sensitive skin syndromes. Eur J Pain 2019; 23: 160-166.

48 Buhé V, Vié K, Guéré C et al. Pathophysiological study of sensitive skin. Acta Derm Venereol 2016; 96: 314-318.

49 Terkelsen AJ, Karlsson P, Lauria G et al. The diagnostic challenge of small fibre neuropathy: clinical presentations, evaluations, and causes. Lancet Neurol 2017; 16: 934-944.

50 Devigili G, Tugnoli V, Penza P et al. The diagnostic criteria for small fibre neuropathy: from symptoms to neuropathology. Brain 2008; 131: $1912-$ 1925.

51 Misery L, Bodéré C, Genestet S et al. Small-fiber neuropathies and skin: news and perspectives for dermatologists. Eur J Dermatol 2013; 24: 147153. 
52 Seidenari S, Francomano M, Mantavoni L. Baseline biophysical parameters in subjects with sensitive skin. Contact Dermatitis 1998; 38: 311-315.

53 Roussaki-Schulze AV, Zafiriou E, Nikoulis D et al. Objective biophysical findings in patients with sensitive skin. Drugs Exp Clin Res 2005; 31 (Suppl): 17-24.

54 Wallengren J. Vasoactive peptides in the skin. J Investig Dermatol Symp Proc 1997; 2: 49-55.

55 Kujala T, Lahti A. Duration of inhibition of non-immunologic immediate contact reactions by acetylsalicylic acid. Contact Dermatitis 1989; 21: 60 61.

56 Verhoeven EW, de Klerk S, Kraaimaat FW et al. Biopsychosocial mechanisms of chronic itch in patients with skin diseases: a review. Acta Derm Venereol 2008; 88: 211-218.

57 Colloca L, Finniss D. Nocebo effects, patient-clinician communication, and therapeutic outcomes. JAMA 2012; 307: 567-568.

58 Schedlowski M, Enck P, Rief W et al. Neuro-bio-behavioral mechanisms of placebo and nocebo responses: implications for clinical trials and clinical practice. Pharmacol Rev 2015; 67: 697-730.

59 Evers AWM, Colloca L, Blease C et al. Implications of placebo and nocebo effects for clinical practice: expert consensus. Psychother Psychosom 2018; 87: 204-210.

60 Evers AWM, Peerdeman KJ, Bartels DJP et al. Placebo and nocebo effects on itch: methodological and clinical implications. In: Misery L, Stander S, eds. Pruritus. Springer, London, 2016.

61 van Laarhoven AIM, Vogelaar ML, Wilder-Smith OH et al. Induction of nocebo and placebo effects on itch and pain by verbal suggestions. Pain 2011; 152: 1486-1494.

62 Stumpf A, Zerey V, Heuft G et al. Itch perception and skin reactions as modulated by verbal suggestions: role of participant's and investigator's sex. Acta Derm Venereol 2016; 96: 619-623.

63 Bartels DJP, van Laarhoven AIM, Haverkamp EA et al. Role of conditioning and verbal suggestion in placebo and nocebo effects on itch. PLoS ONE 2014; 9: e91727.

64 Liccardi G, Senna G, Russo M et al. Evaluation of the nocebo effect during oral challenge in patients with adverse drug reactions. J Investig Allergol Clin Immunol 2004; 14: 104-107.

65 Bernard A, Ficheux AS, Nedelec AS et al. Induction of sensitive skin and sensitive scalp by hair dyeing. Int J Eng Res Gen Sci 2016; 4: 5.

66 Kerscher M. Principles of treatment and protection for sensitive skin. Hautarzt 2011; 62: 906-913.

67 Giménez-Arnau A. Standards for the protection of skin barrier function. Curr Probl Dermatol 2016; 49: 123-124.

68 Simon D, Nobbe S, Nägeli M et al. Short- and long-term effects of two emollients on itching and skin restoration in xerotic eczema. Dermatol Ther 2018; 2018: e1269.
69 Berardesca E, Mortillo S, Cameli N et al. Efficacy of a shower cream and a lotion with skin-identical lipids in healthy subjects with atopic dry skin. J Cosmet Dermatol 2018; 17: 477-483.

70 Weisshaar E. Saving the barrier by prevention. Curr Probl Ddermatol 2016; 49: 152-158.

71 D'Orazio J, Jarrett S, Amaro-Ortiz A et al. UV radiation and the skin. Int J Mol Sci 2013; 14: 12222-12248.

72 Dieamant Gde C, Velazquez Pereda Mdel C, Eberlin S et al. Neuroimmunomodulatory compound for sensitive skin care: in vitro and clinical assessment. J Cosmet Dermatol 2008; 7: 112-119.

73 Lopes DM, McMahon SB. Ultraviolet radiation on the skin: a painful experience? CNS Neurosci Ther 2016; 22: 118-126.

74 Moyal DD, Fourtanier AM. Broad-spectrum sunscreens provide better protection from solar ultraviolet-simulated radiation and natural sunlight-induced immunosuppression in human beings. J Am Acad Dermatol 2008; 58(Suppl 2): S149-S154.

75 Reich A, Harupa M, Bury M et al. Application of sunscreen preparations: a need to change the regulations. Photodermatol Photoimmunol Photomed 2009; 25: 242-244.

76 Misery L. Neuropsychiatric factors in sensitive skin. Clin Dermatol 2017; 35: 281-284.

77 Bodó E, Kovács I, Telek A et al. Vanilloid receptor-1 (VR1) is widely expressed on various epithelial and mesenchymal cell types of human skin. J Invest Dermatol 2004; 123: 410-413.

78 Kueper T, Krohn M, Haustedt LO et al. Inhibition of TRPV1 for the treatment of sensitive skin. Exp Dermatol 2010; 19: 980-986.

79 Fauger A, Lhoste A, Chavagnac-Bonneville $\mathrm{M}$ et al. Effects of a new topical combination on sensitive skin. J Cosmet Sci 2015; 66: 79-86.

80 Choi M, Kim JE, Cho KH et al. In vivo and in vitro analysis of low level light therapy: a useful therapeutic approach for sensitive skin. Lasers Med Sci 2013; 28: 1573-1579.

81 Sonbol H, Brenaut E, Nowak EML. Efficacy and tolerance tolerability of the phototherapy by with Light Emitting Diodelight-emitting diodes for sensitive skin: a pilot study. Front Med. in revision

82 Draelos ZD, Levy SB, Lutrario C et al. Evaluation of the performance of a nature-based sensitive skin regimen in subjects with clinically diagnosed sensitive skin. J Drugs Dermatol 2018; 17: 908-913.

83 Evers AWM. Using the placebo effect: how expectations and learned immune function can optimize dermatological treatments. Exp Dermatol 2017; 26: 18-21.

84 Colagiuri B, Schenk LA, Kessler MD et al. The placebo effect: from concepts to genes. Neuroscience 2015; 307: 171-190.

85 Meeuwis SH, Van Middendorp H, Veldhuijzen DS et al. Placebo effects of open-label verbal suggestions on itch. Acta Derm Venereol 2015; 98: 268-274. 